\title{
Mode transition of interacting buoyant non-premixed flames
}

\author{
Anek BUNKWANG*,**, Tsuneyoshi MATSUOKA* and Yuji NAKAMURA* \\ *Department of Mechanical Engineering, Toyohashi University of Technology \\ 1-1 Hibarigaoka, Tempaku, Toyohashi, 441-8580, Japan \\ E-mail: yuji@me.tut.ac.jp \\ **Department of Mechatronics Engineering, Pathumwan Institute of Technology \\ 833 Wangmai, Pathumwan, Bangkok, 10330, Thailand
}

Received: 26 December 2019; Revised: 30 January 2020; Accepted: 6 February 2020

\begin{abstract}
The dynamic behavior, especially in the transition to oscillation mode (in-phase and anti-phase), of two interacting non-premixed methane-air jet flames was investigated experimentally. A well-controllable experimental system for the present purpose was constructed and key parameters; such as fuel flowrate $(Q)$, burner diameter $(d)$, and burner separation distance $(L)$, were varied systematically. A well-known periodic motion of the flame was observed and the frequency monitored by thermocouples mounted adjacent to the burner exit. Time-variation of flame shape was recorded by a high speed camera associated with the optical imaging visualization. It was found that the flickering frequency was insensitive to the fuel flowrate, $Q$, implying that inertia played secondary role in the transition. Instead, the burner critical separation distance for the transition $\left(L_{c r t}\right)$ varied when various burner diameters were used, confirming that the difference in distance played an important role in the transition. It was found that the critical condition could be summarized by an updated correlation as $d \times L_{c r t}^{3} \sim$ const. This is slightly different from the one recently proposed by Yang et al. (2019), which was given under a narrower range of the fire scale. Accordingly, the critical condition can also be described by the critical value of the updated global parameter, such as $\alpha^{3} \mathrm{Gr}^{4 / 3}$, where $\alpha$ and $\mathrm{Gr}$ denote the length ratio $\left(L_{c r t} / d\right)$ and Grashof number based on the burner diameter, respectively.
\end{abstract}

Keywords : Flickering, Non-premixed flame, Interaction, Transition, Grashof number

\section{Introduction}

It is well-known that a non-premixed flame over a certain size oscillates under a gravimetric field, especially on the upper part, with the periodicity motion of a pinched off flame pocket (Hottel and Hawthorne, 1949; Carpio et al., 2012). Identical oscillation features can be found irrespective of the source of the flame (jet flame, pool fire etc.), emphasizing that the dynamic behaviors are controlled by the buoyancy. These periodic dynamics have been called "flame flickering (or flame puffing)" and the frequency is an important characteristic, as well as the quantity.

Previous studies (Hamins et al., 1992; Cetegen and Ahmed, 1993; Cetegen and Dong, 2000), have confirmed the prominent scaling relation, $f \sim d^{-1 / 2}$, in a wide range of fire scales $(d)$ irrespective of the kinds of fuel. Cetegen and Ahmed (1993) developed a mathematical model to predict the dynamic frequency based on the convective time scale of flame flickers. Since the main convective flow is induced by the buoyancy, the burner scale acts as representative scale to control the magnitude of buoyancy-induced flow velocity. From a fluid dynamics point of view, the buoyancy-induced flow forms the shedding toroidal vortex along the flame. In this way, the relation between the fire scale and dynamic frequency of the flame is correlated as described. Most recently, Xia et al. (2018) introduced vortex-dynamical principles and attempted to observe the relation between flame and vortex dynamics. According to their work, the total vorticity (circulation) of the toroidal vortex shows independency on the geometric shape of burner port; rather it is dictated by the vertical length of vortex sheet. This is consistent with the experimental observation that the pinching-off length of the flame is nearly identical.

When two buoyant flames are positioned close to each other, the interaction of inner-side shear layer, where the 
toroidal vortex occurs between the two flames, shows a unique feature of global flame dynamics. Indeed, an interesting yet strange flame dynamic behavior is dependent on the distance between the flames. The pioneering work on this subject appeared in the mid-60s from an MIT group (Toong et al., 1965). When the flames are separated at a short distance, both flames flicker at an identical phase (in-phase oscillation mode) at a nearly constant frequency, whereas they exhibit an anti-phase oscillation mode when the flames are apart at a certain distance. The frequency decreases with the distance. Interestingly, there is clear jump in the distance between the flames at which the oscillation mode changes one to the other. In terms of the presence of two modes, Kitahara et al. (2009) developed a simple mathematical model including a non-linear effect via radiation energy transfer, and revealed that such a non-linear effect plays a key role on switching to oscillation mode. Later, Mochizuki and Nakamura (2015) and Mochizuki et al. (2017) developed a 3-D numerical model for interacting two jet burners and showed that the radiation effect did not play the primary role in the appearance of two modes. Together with optical imaging technique (Bunkwang et al., 2019), it was confirmed that the fluid dynamics induced by buoyancy played a key role in the appearance of two modes and their transition. Yang et al. (2019) performed 3-D numerical simulation for pool fires and proposed that the transition can be summarized by a modified Grashof number. Although their model successfully revealed the simple relation between the fire scale ( $d$ : pool/burner (inner) diameter) and separation distance $(L)$ as $d \times L^{2} \sim$ const. at the transition, their working range was limited, for instance, the range of the burner scale (less than factor of 3 ) because the target phenomena was known as highly non-linear one and the actual trend to be investigated needed a wide range, at least a factor of 5 or higher. Thus, the critical conditions based on Yang's model have been given as a "range", not "value", suggesting that the precision of the proposed strategy needs to be validated further. It is not easy to establish a precise experiment for a larger burner because the burner exhibits a random fluctuation of the flame and measurement of the exact transition point is quite challenging. This study attempts to provide precise data on the transition, which can be used for further validation on the transition model.

The present study attempted to study the mode transition experimentally by varying three important system parameters of this phenomenon: fuel flowrate $(Q)$; burner diameter $(d)$; and burner separation distance $(L)$. Our experimental system was upgraded to improve repeatability even for the larger burner case to enable validation of Yang's model. Introducing careful and precise measurement techniques, we successfully showed a trend in a wide range of parameters and upgraded the correlation between the adopted burner diameter and critical burner separation distance at the transition.

\section{Methodologies \\ 2.1 Experimental apparatus}

The experimental apparatus used in this work is shown in Fig. 1. It is basically the same as our previous work (Mochizuki and Nakamura, 2015; Mochizuki et al., 2017; Bunkwang et al., 2019). All experiments were performed at in the open, in the lab. The experiment was repeated for least twice. Sample standard deviation was adopted for uncertainty. Two identical round-shaped burners were located vertically and research grade methane gas (purity: $99.4 \%$ ) was issued upward at identical flowrate, $Q$ [cc/min]. Flowrate of the methane was controlled by a hand-made flow-control system, which consisted of a monometer with the accuracy approximately higher than $99 \%$. One burner was fixed at to the floor and the other burner mounted on an optical stage, which enabled us to modify the burner separation distance, $L$, within a range of $0.0-100.0 \mathrm{~mm}$. The condition $L=0 \mathrm{~mm}$ indicated two burners were perfectly placed in parallel, in other words, the burner axes are at the distance of burner diameter $(d)$. Multi-layered mesh screens surrounded the apparatus in order to eliminate any disturbance from the ambient to the flame dynamics. Ignition was made using a burner torch, and the system allowed to stabilize before recording data.

Dynamical flame behavior was recorded by a high-speed camera (CASIO EX-F1: 300 fps with 512 x 384 pixels). As shown in Fig. 1 the camera was only located at front. Additionally, we obtained the orthogonal photography in order to check whether the flame moved in front-back direction or not during the interaction event. Fine thermocouples (TCs: K-type, junction dia.: $0.25 \mathrm{~mm}$ ) were equipped near the burner exit to record the time-variation of field temperature signal via data logger (Graphtec GL-900) at the sampling rate of 10 millisecond. Harmonized frequencies, $f$ [Hz], were obtained by analyzing the TCs' signal by operating the Fast Fourier transformation (FFT). Investigation is performed under the condition of $F r<1$, suggesting that the buoyancy-controlling flame (note that Froude number is defined as jet ejecting velocity, burner diameter and gravity acceleration). The range of fuel flowrate $(Q)$, burner diameter $(d)$, and 
separation distance $(L)$, varied in this study is $Q \leq 3000 \mathrm{cc} / \mathrm{min}, d \leq 55 \mathrm{~mm}$ and $0 \leq L \leq 100 \mathrm{~mm}$, respectively.

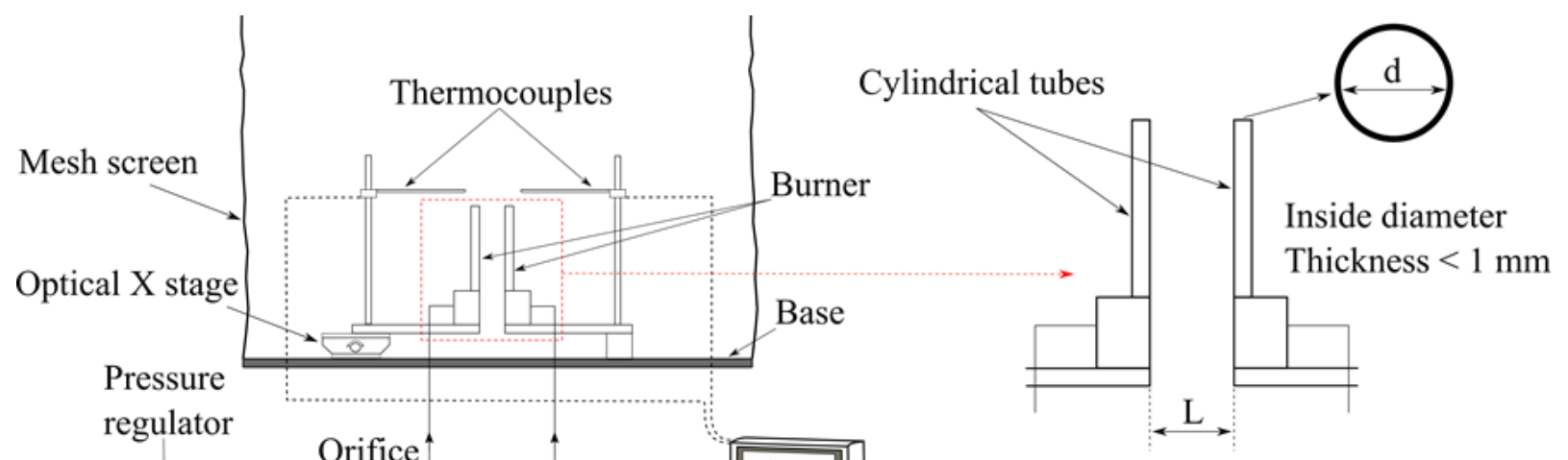

Burner separation distance

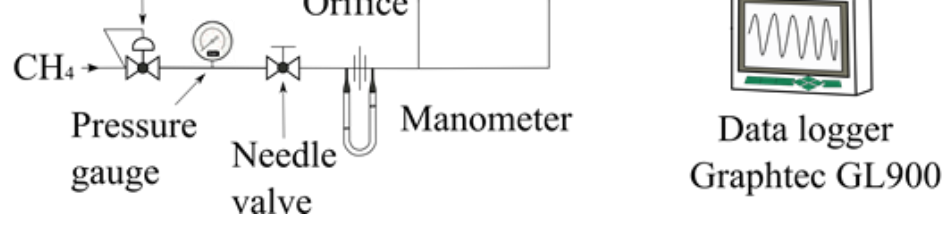

Fig. 1 Schematics of experimental apparatus.

\subsection{Schlieren imaging}

To visualize the formation of the hot plume surrounded by the visible flame, Schlieren imaging technique was used (Bunkwang et al., 2019). Apparatus and representative visualized images are shown in Fig. 2. The visualized target (i.e. flames) was positioned between two collimating mirrors, with spacing distance set at approximately $5 \mathrm{~m}$. A stroboscope (Types: MSX-1A, Metered variac: 0 - 240V, Flashing range: 100 - 26000 rpm, Flashlamps: xenon) was used as a lightsource. The focal length and diameter of the mirrors were $3.5 \mathrm{~m}$ and $0.28 \mathrm{~m}$ respectively. The knife-edge and light source were placed at the focusing point of the parabolic mirror and the high speed camera on a tripod was positioned close using a $300 \mathrm{~mm}$ zoom lens to fill the image plane. The spatial resolution was approximately $0.58 \mathrm{~mm} /$ pixel in the imaging plate. Unrefracted light rays were blocked by a cut-off implement, resulting in the gray pixel-intensity level of the background. Adjusting the shoe system, a clear visualization of the both direct flame and hot plume was available to help to understand how the flame start to flicker.

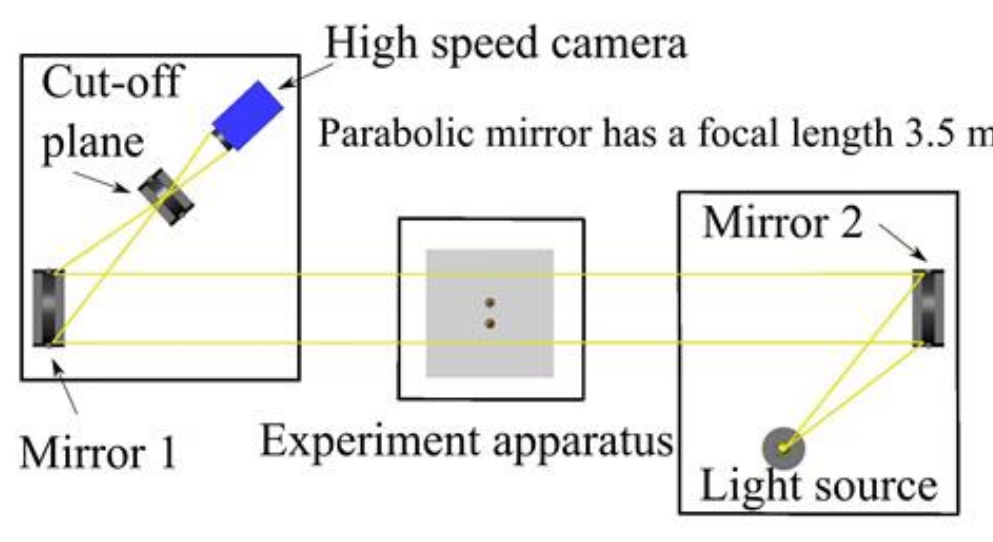

(a) Opitical setup

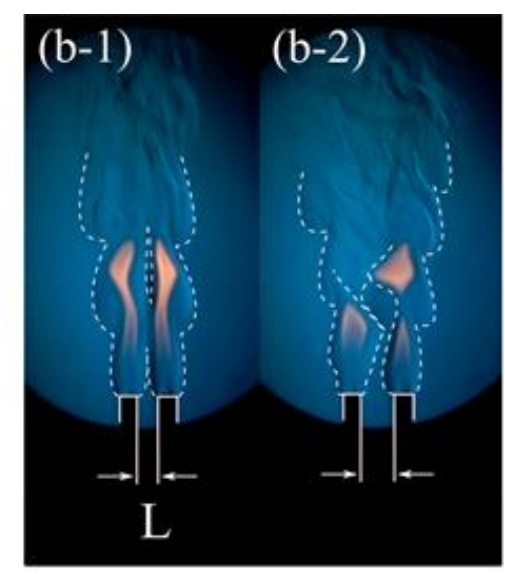

(b) Typical image

Fig. 2 Schlieren optical imaging system (a) and representative images observed at $L=15 \mathrm{~mm}$ (in-phase) and $25 \mathrm{~mm}$ (anti-phase), respectively (b). Broken lines in (b) indicates the representative boundary of thermal layer. 


\section{Experimental results}

\subsection{Oscillation mode of interacting flames and its transition}

Figure 3 shows typical sequential pictures (front directional and orthogonal photography) of the pulsating methaneair jet flames obtained, with prescribed fuel flowrate $(Q)$ and burner separation distance $(L)$, together with the corresponding Schlieren images. Previous studies (e.g., Toong et al., 1965; Kitahara et al., 2009; Mochizuki and Nakamura, 2015; Mochizuki et al., 2017) identified two kinds of dynamic modes exhibited by the interacting flames, such as in-phase ((a)-(c)) and anti-phase ((d)-(f)) modes, depending on the adopted condition. As seen in the figure, two buoyant flames successfully synchronized and a solid periodic motion was attained.
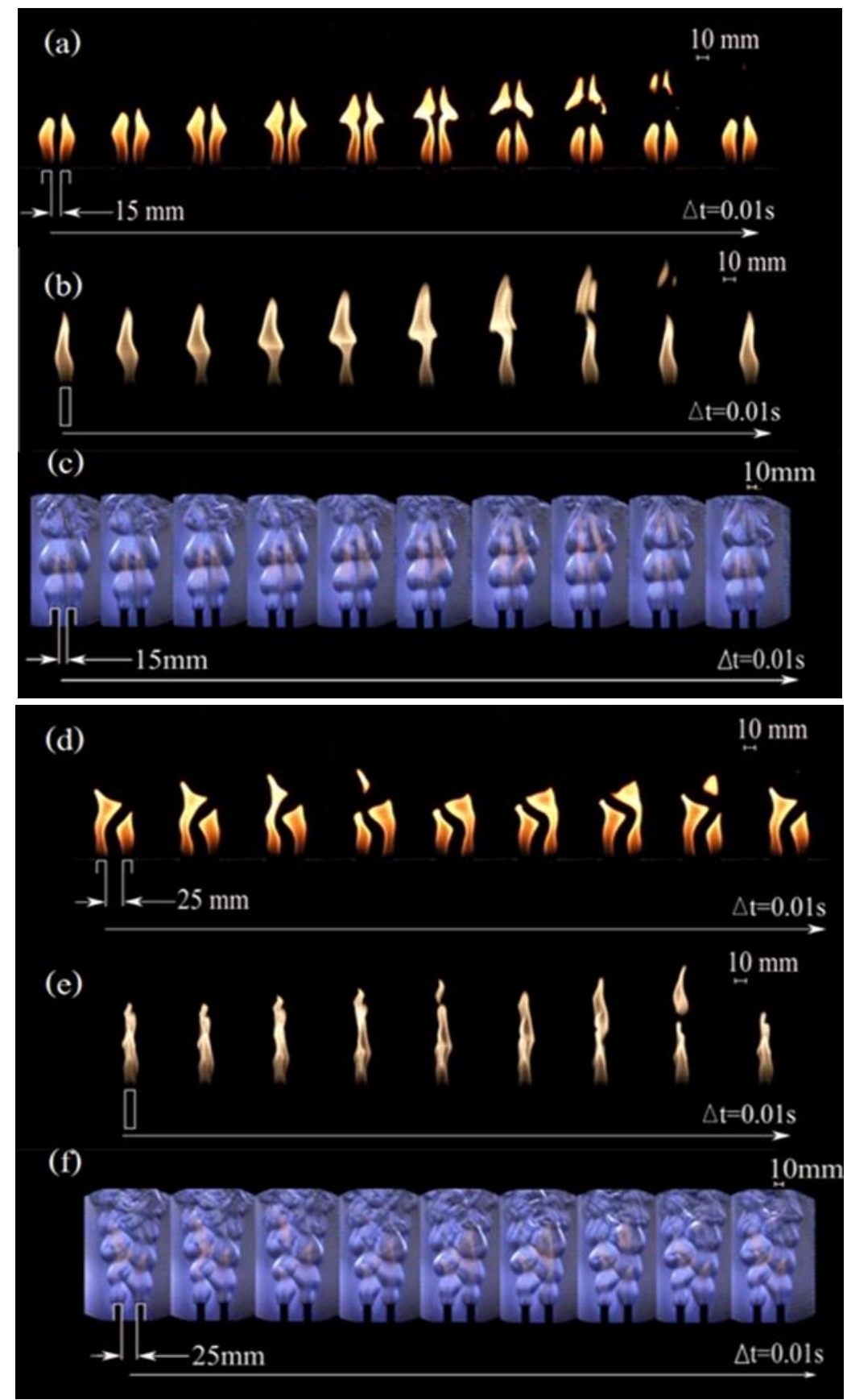

Fig. 3 Time-sequential images of pulsating methane plume at in-/anti-phase mode: direct photography (a)(d), orthogonal photography (b)(e) and Schlieren images (c)(f). (a)-(c) show in-phase oscillation, $L=15 \mathrm{~mm}, d$ $=10 \mathrm{~mm}, Q=1000 \mathrm{cc} / \mathrm{min}$. (d)-(f) show anti-phase oscillation, $L=25 \mathrm{~mm}, d=10 \mathrm{~mm}, Q=1000 \mathrm{cc} / \mathrm{min}$. Time interval $(\Delta \mathrm{t})$ is $0.01 \mathrm{~s}$. 
Based on the orthogonal images shown in $(b)(e)$, pinched off flames at in-phase mode were relatively thicker than in the anti-phase mode. Nevertheless, it was identified that the bottom part of flames for both modes did not move frontto-back, suggesting that the two-dimensional observation of the flickering phenomena is enough to grasp the overall motion of the flames. In this regard, Schlieren imaging from front view delivered beneficial yet sufficient information of flame dynamics. From Fig. 3(c), it can be understood that the hot plume over the flame fully developed and merged when in-phase mode was attained, then the large hot plume could drive the whole dynamics of the flame. However, when the anti-phase mode was attained as shown in Fig. 3(f), hot plumes made by each jet flame did not merge completely, but rather, were always separated at the flaming zone although they were disturbed and mixed far downstream.

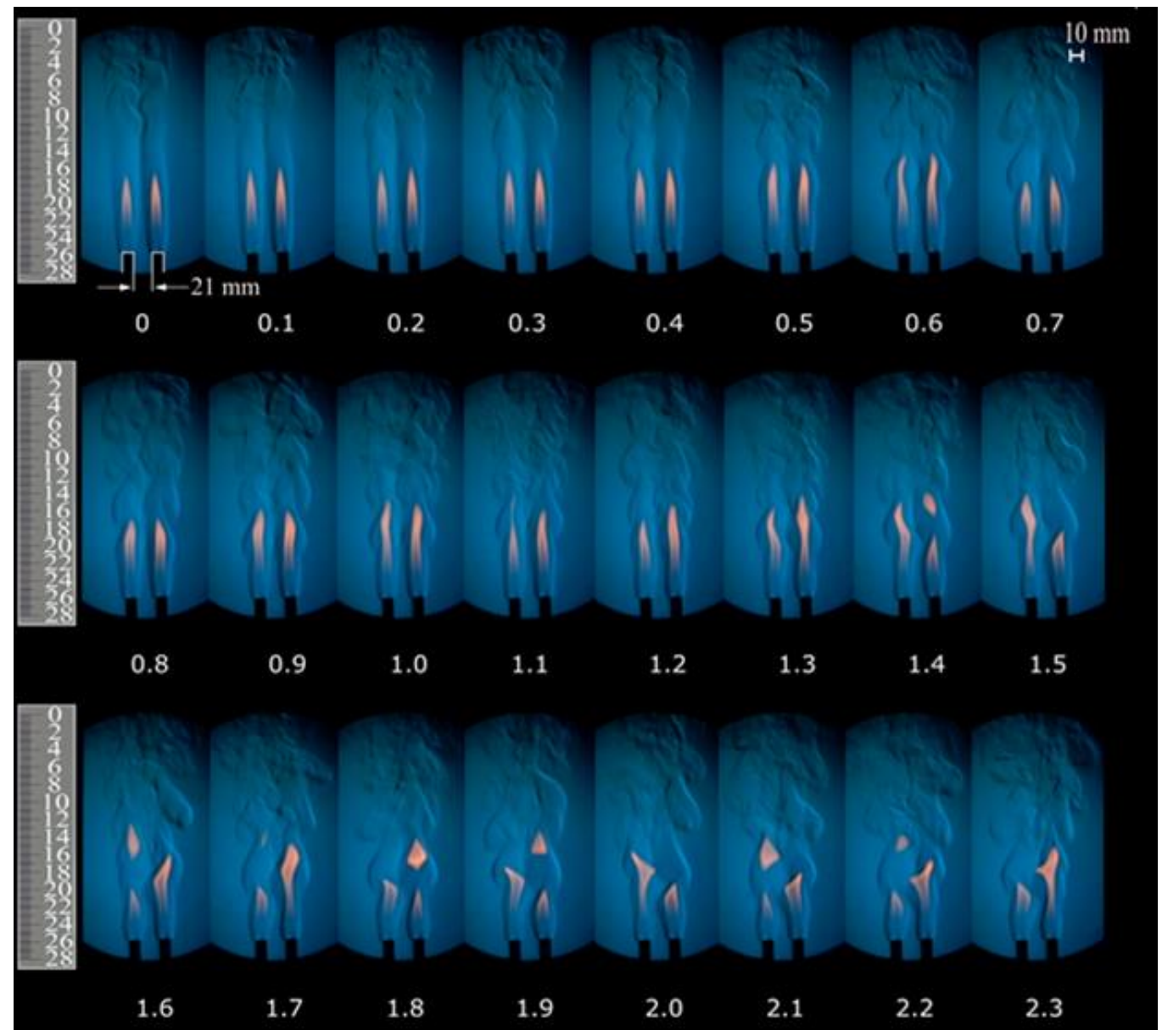

Fig. 4 Time-sequential images of pulsating methane plume at transition condition: $L=21 \mathrm{~mm}, d=10 \mathrm{~mm}, Q=500$ $\mathrm{cc} / \mathrm{min}$. Time interval $(\Delta \mathrm{t})$ is $0.01 \mathrm{~s}$.

In Fig. 4, time-sequential Schlieren images in a transient condition are shown. In this figure, a series of images of the transition from weak flickering motion to in-phase and anti-phase oscillation are presented. Numbers shown in the images indicate the time (sec.) at intervals of $0.1 \mathrm{sec}$. Because the typical flickering frequency is $10-15 \mathrm{~Hz}$, using this size of burner with $0.1 \mathrm{sec}$ interval images it is not possible to track the complete motion of the flickering. The main purpose of Fig. 4 is how the hot plume interacts with the flame in time. At an early stage $(<0.4 \mathrm{sec})$, the hot plume around the flame is rather smooth and forms a steady boundary layer. Far downstream, however, the disturbance is identified due to the buoyancy acceleration, and forms a shear stress along the hot plume boundary. As time progresses, the disturbance front travels toward the upstream and eventually reaches the upper part of the flame at 0.6 sec, to promote the deformation of the visible flame. Till $1.0 \mathrm{sec}$, symmetry oscillation (namely in-phase mode) is presented, showing that two flames oscillate in the same way. However, interestingly, the left and right flames give slight asymmetry at 1.1 sec, then the asymmetry grows in time to achieve the transition to anti-phase mode. Once anti-phase mode is achieved, the flames prefer to stay in that mode and is difficult to reform to the in-phase oscillation. From this observation, as 
previously predicted (Mochizuki et al., 2017), anti-phase mode (flame exhibits asymmetry) seems more stable than inphase mode.

The results showed that the behavior of the hot plume is important for triggering the two kinds of transition observed here (from no motion to in-phase, and from in-phase to anti-phase). Hence, it is expected that the promotion of buoyantflow intensity may result in modifying the transition condition.

\subsection{Oscillation frequency: Modifying of the experimental parameters $Q, d, L$}

Time variation of signal from thermocouples (TCs) fixed adjacent to the flame base were analyzed to identify characteristics of the dynamic resonance behavior. Fig. 5 shows a representative example of the characteristic frequency obtained by Fast Fourier Transform (FFT) of TC signals, with an apparent peak at a certain frequency, ensuring the dynamic motion shall be characterized by that frequency. Frequency stays nearly constant when the in-phase mode of dynamics is experienced, whereas it decays when the anti-phase mode of dynamics is experienced. The transition from one mode to the other occurs around $20 \mathrm{~mm}$ of the burner separation distance, defined as the "critical condition" for mode transition. It is interesting to note that uncertainty at the critical condition becomes extra-ordinarily large compared to the other condition studied. This is due to various modes that randomly appear, including two dynamic modes (inphase and anti-phase), or even stationary (no motion), suggesting that this mode is quite unstable. Hereafter, the critical burner separation distance is denoted as $L_{c r t}$. As the response of frequency $(f)$ depending on the separation distance $(L)$ provides the characteristics of the oscillation behavior presented in this system, we investigated $f$ - $L$ curve with various $Q$ and $d$. Note that $L_{c r t}$ is theoretically solely determined, however, there is certain (even narrow) range as shown in Fig. 5. Thus, in this study, $L_{c r t}$ is defined as the center value of transition range as shown in Fig. 5.

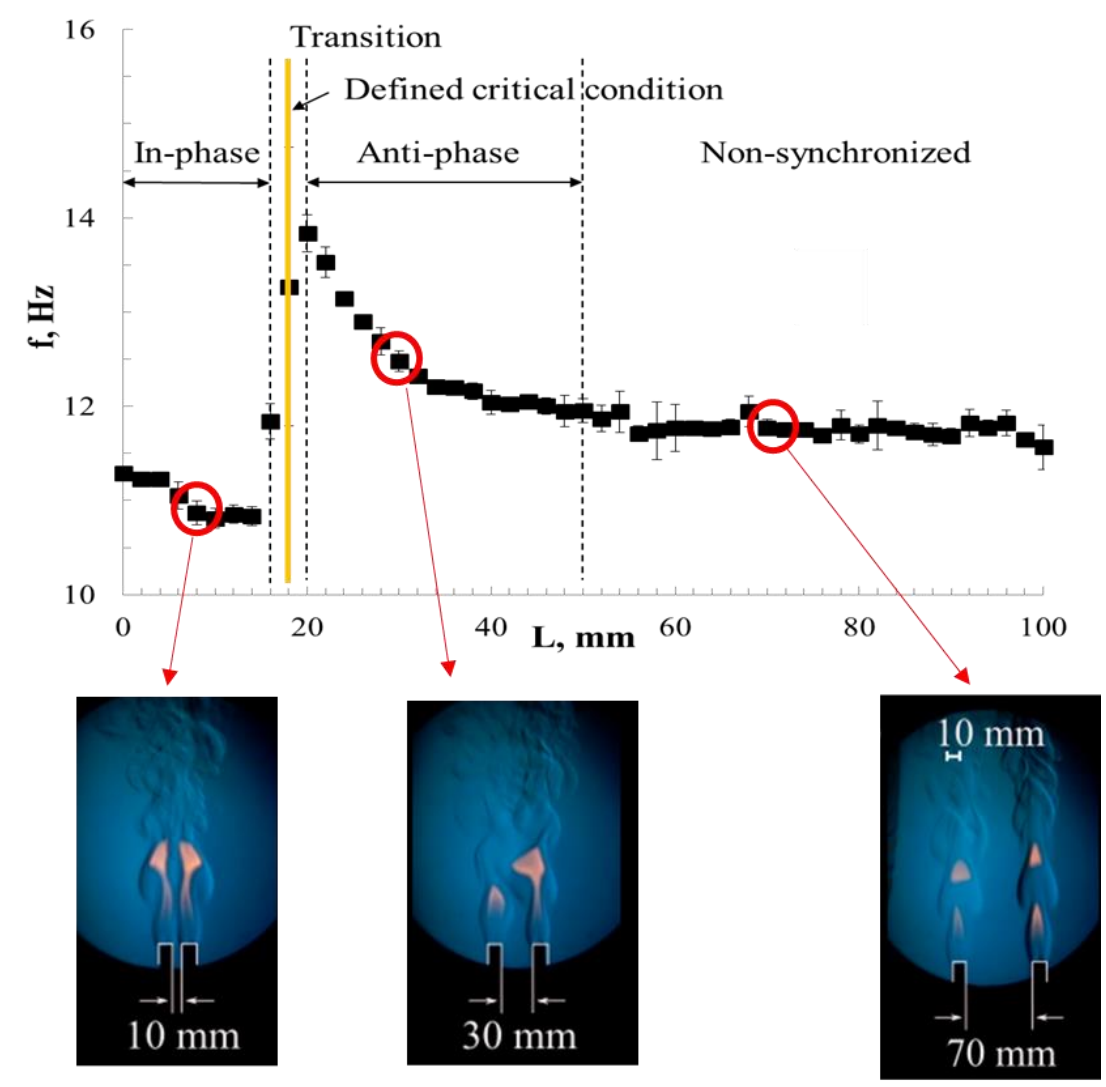

Fig. 5 Effect of burner separation distance on flickering frequency and each resonance dynamic modes $(d=10 \mathrm{~mm}$, $Q=500 \mathrm{cc} / \mathrm{min}$ ). 


\subsection{Effect of fuel flowrate $(Q)$ on overall oscillation behavior $(f)$}

Figure 6 (a)-(c) show typical Schlieren images at various fuel flowrates $(Q=500 \mathrm{cc} / \mathrm{min}, 600 \mathrm{cc} / \mathrm{min}, 700 \mathrm{cc} / \mathrm{min}$, respectively). Fig. 6 (d) summarizes the averaged flickering frequency $(f)$, which is considered as the characteristic dynamic measure of the present system, vs. the burner separation distance, $L$ for $d=10 \mathrm{~mm}$ (smaller burner case). In Fig. 6 (d), four cases of fuel flowrate ranging from 500 to $3500 \mathrm{cc} / \mathrm{min}$ are shown for direct comparison.

From Figs. 6 (a)-(c), a trend for flame height to increase as fuel flowrate increased was found. Further, the shape and the scale of hot-plume above the flame did not significantly differ under different flowrates. We deduced that the length of the bottom stem seemed to increase, which is most responsible for the increase of the flame height. In this regard, the fluctuation is only presented at the top part of the flame as well as the hot plume.
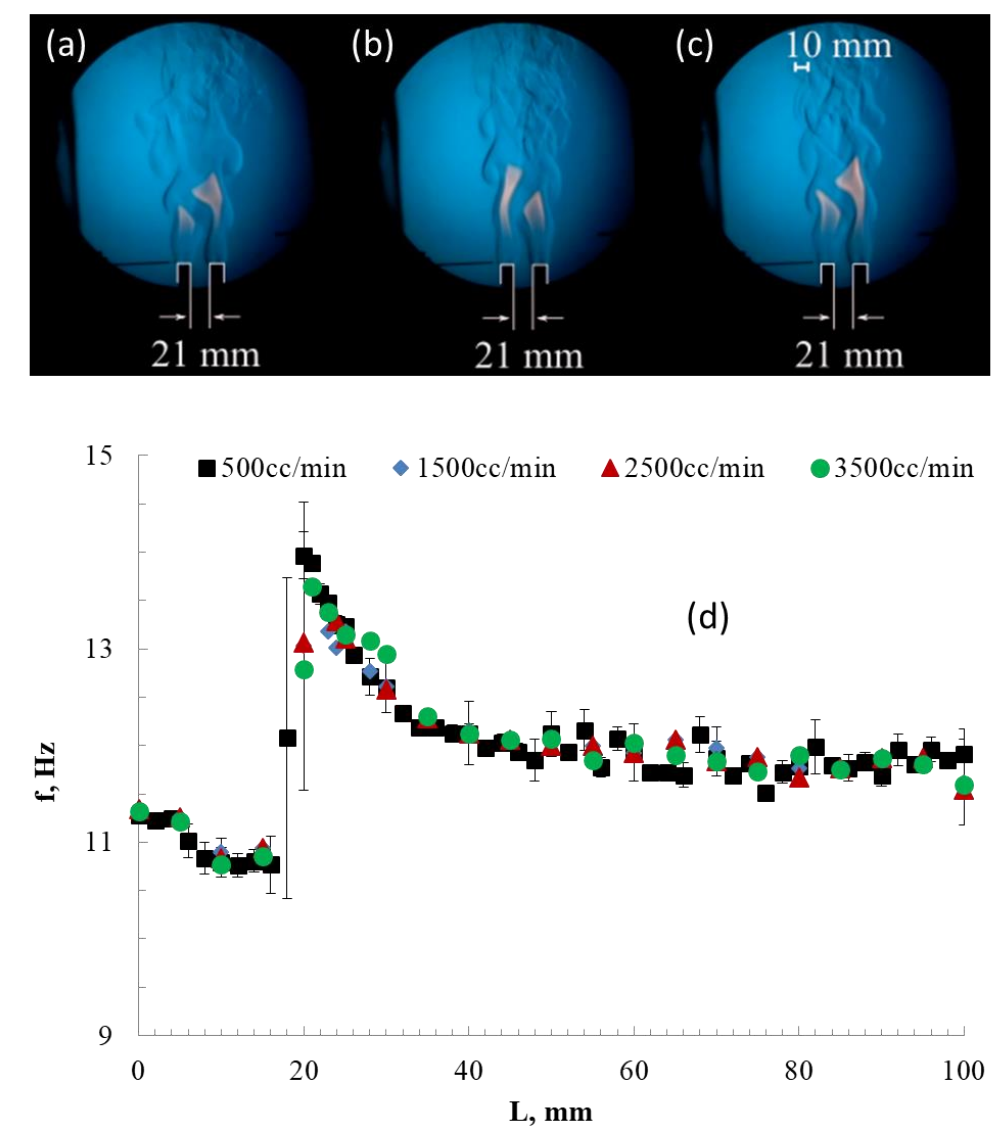

Fig. 6 Typical Schlieren images at various fuel flowrates (a): $Q=500 \mathrm{cc} / \mathrm{min},(\mathrm{b}): Q=600 \mathrm{cc} / \mathrm{min},(\mathrm{c}): Q=700$ $\mathrm{cc} / \mathrm{min}$, and (d) Effect of burner separation distance on flickering frequency for varying of fuel flowrate. All cases shown here are at burner diameter of $d=10 \mathrm{~mm}$.

In Fig. 6 (d), an abrupt frequency jump was clearly identified at the critical separation distance $\left(L_{\text {crt }}\right)$, nearly $20 \mathrm{~mm}$ for the wide range of the flowrate. More importantly, not only the jump condition (at transition), but also the overall dependency of frequency on the separation distance was nearly identical. This indicated that the periodic motion of the flame was quite insensitive to the jet momentum, ensuring that the periodical dynamic behavior observed in this study was mainly governed by the buoyancy, not the inertia (momentum) of the fuel flow jet.

\subsection{Effect of burner diameter $(d)$ on overall oscillation behavior $(f)$}

Figure 7 (a)-(c) show the typical Schlieren images at various burner diameters $(d=10 \mathrm{~mm}, 18 \mathrm{~mm}, 30 \mathrm{~mm}$, 
respectively, with $Q=900 \mathrm{cc} / \mathrm{min}$ ) and (d) summarizes the averaged flickering frequency ( $f$ ), which is considered as the characteristic dynamic measure of the present system, vs. the burner separation distance, $L$. The adopted fuel flowrate in Fig. 7 (d) is $Q=1000 \mathrm{cc} / \mathrm{min}$ for smaller burner cases $(d=10,18$, and $30 \mathrm{~mm})$, whereas $Q=2000 \mathrm{cc} / \mathrm{min}$ and 3000 $\mathrm{cc} / \mathrm{min}$ for larger burner cases $(d=45$ and $55 \mathrm{~mm}$, respectively). Because a variation of $Q$ does not have a significant effect on $f$ - $L$ plane, as confirmed in the previous section (see Fig. 6), applying various $Q$ values to investigate the effect of $D$ on flickering frequency, $f$, is not really needed. In Fig. 7 (d), all five cases of burner diameters from $d=10 \mathrm{~mm}$ to $55 \mathrm{~mm}$ are shown for direct comparison.

Figs. 7 (a)-(c) show the neck part (eventually pinched off) gradually moving upstream (close to the burner surface) as the burner diameter increases. Obviously, the shape and scale of the hot plume above the flame are not constant, but increase as burner diameter increases. Because the gap size is the same for three cases, an interaction of thermal boundary layers formed around each flame as seen earlier for larger burner cases. This suggests that the critical transient condition for separation distance $\left(L_{c r t}\right)$ is affected when the burner diameter is varied.
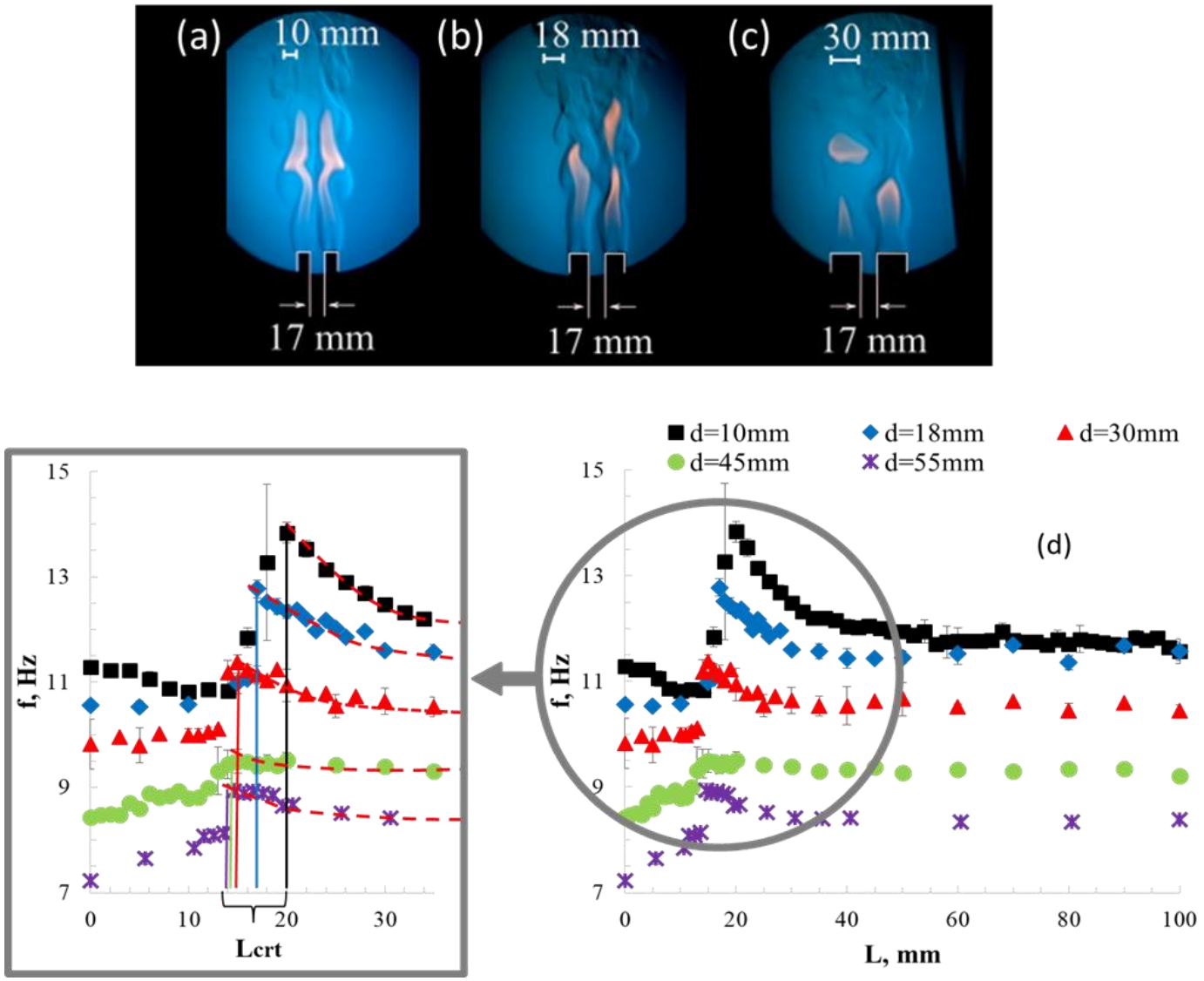

Fig. 7 A typical Schlieren images at various burner diameter (above): $d=10 \mathrm{~mm},(\mathrm{~b}): d=18 \mathrm{~mm},(\mathrm{c}): d=30 \mathrm{~mm}$, with constant flowrate at $Q=900 \mathrm{cc} / \mathrm{min}$. Effect of burner separation distance on flickering frequency for varying of fuel flowrate (bottom). Cases $(d=10,18$ and $30 \mathrm{~mm})$ shown here are at the constant fuel flowrate at $Q=1000 \mathrm{cc} / \mathrm{min}$, but $d=45$ and $55 \mathrm{~mm}$ of 2000 and $3000 \mathrm{cc} / \mathrm{min}$.

In Fig. 7 (d), an abrupt frequency jump can be clearly identified at the critical separation distance $\left(L_{c r t}\right)$ for all tested cases of the burner diameter. Frequency jump-gap before/after at the transition becomes smaller as the burner diameter increases, suggesting the difference between in-phase and anti-phase is no longer clear enough, and the direct flame images tend to give a highly fluctuated appearance (unlike with smaller diameter as shown in Fig. 3). This confirms that comparisons with $f-L$ plane is a solid strategy for examining the characteristics of oscillation behavior. Nevertheless, it is necessary to find a similar trend in $f$ - $L$ plane for all tested burner cases. Most importantly, as noted in Fig. 7 (d), there is a clear dependency of $L_{c r t}$ on the applied burner diameter, for instance, a larger diameter gives a smaller $L_{c r t}$, under 
the conditions studied here, confirming that there are two important length scales $\left(d\right.$ and $\left.L_{c r t}\right)$ in the present system when the mode transition is being studied.

Interestingly, these two important scales have clear a dependency, as summarized in Fig. $8, d \sim L_{c r t}^{-3}$ (namely, $d \times$ $L_{c r t}^{3} \sim$ const.). The predicted values (three plots) by Yang et al. (2019) are also embedded in the figure, showing their plots are well-fitted to the trend obtained by the present experiment. With this respect, our experimental work for wider a range of fire scale $(d)$ may reveal a "universal" trend for transition behavior.

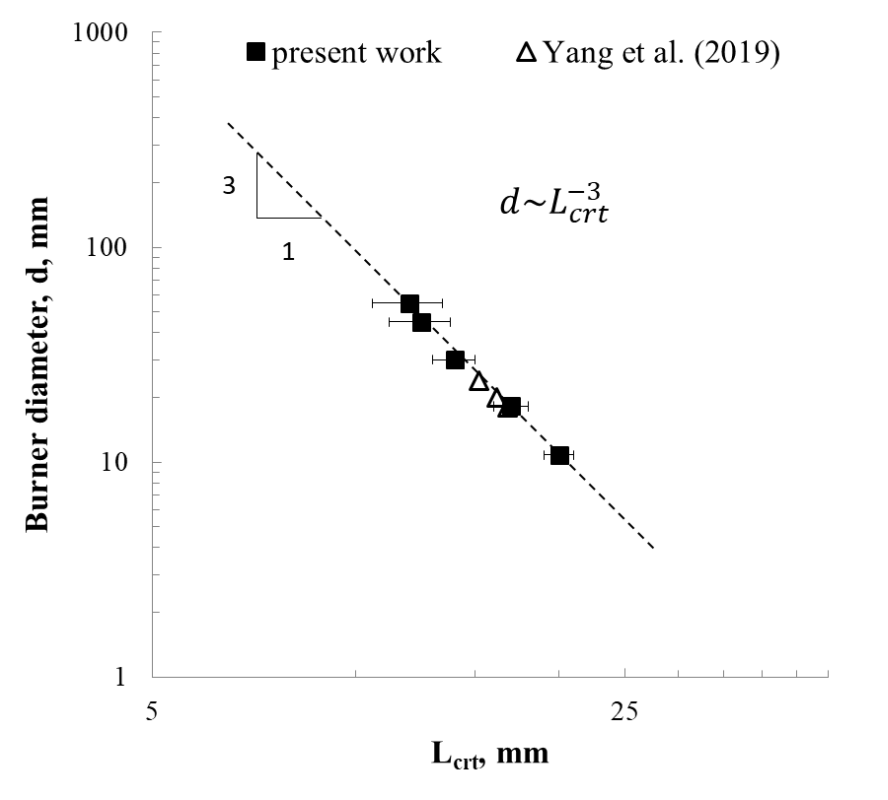

Fig. 8 Relation between $L_{c r t}$ and $d$ based on the measured data in this study. Predicted values by Yang et al. (2019) are also embedded in the figure.

\section{Updating the mode transition model}

When discussing the update modeling on the mode transition behavior of the two flickering flames, there are several assumptions to apply. (1) The flame sheet model can be adopted (we can assume that Damkohler number is infinity, suggesting that the transition phenomena is far from either ignition/extinction); (2) Lewis number is unity; (3) the presence of a burner plays a minor role on overall flame dynamics (namely, the boundary layer formed by the burner is not really important; (4) radiation does not have a primary role in flame dynamics (see Mochizuki et al., 2017). Further, Grashof number $\left(G r=g d^{3} / v_{a}^{2} ; g\right.$ is gravity acceleration, $v_{a}$ is kinematic viscosity of air $)$ is the only responsible nondimensional number to describe the dynamics in the present system. In addition, we have already learned from Fig. 7 that there are two important length scales in this system, $L$ and $d$. Hence, we should consider their ratio $(\alpha=L / d)$ as the additional non-dimensional number to characterize the target phenomena. Finally, the characteristic parameter to govern flame behavior in the present system (including the transition), say $\Lambda$, shall be described as the combination of two nondimensional groups as below:

$$
\Lambda \sim \alpha^{n} G r^{m}=[L / d]^{n}\left[g d^{3} / v_{a}^{2}\right]^{m}
$$

To fulfill the observation, as confirmed in Fig. 8, the exponent of $\mathrm{n}$ and $\mathrm{m}$ must be $n=3, m=4 / 3$. Namely,

$$
\Lambda_{c r t} \sim \alpha^{3} G r^{4 / 3}=\left[L_{c r t} / d\right]^{3}\left[g d^{3} / v_{a}^{2}\right]^{4 / 3}
$$

Suppose the buoyancy-induced flow, $U_{b}$, is scaled as $U_{b} \sim \sqrt{g d}$ under the assumption of the unity of the Froude number, $\Lambda_{c r t}$ can be rewritten as follows using Reynolds number based on the buoyancy-induced flow and the burner 
separation distance at transition $\left(R e_{L}\right)$.

$$
\Lambda_{c r t} \sim\left[L_{c r t} / d\right]^{3}\left[U_{b}^{2} d^{2} / v_{a}^{2}\right]^{4 / 3}=\left[L_{c r t} / d\right]^{1 / 3}\left[U_{b}^{2} L_{c r t}^{2} / v_{a}^{2}\right]^{4 / 3}=\alpha^{1 / 3} R e_{L}^{8 / 3}
$$

This Reynolds number is not the one to characterize the jet flow but the one to characterize the transport between two burners.
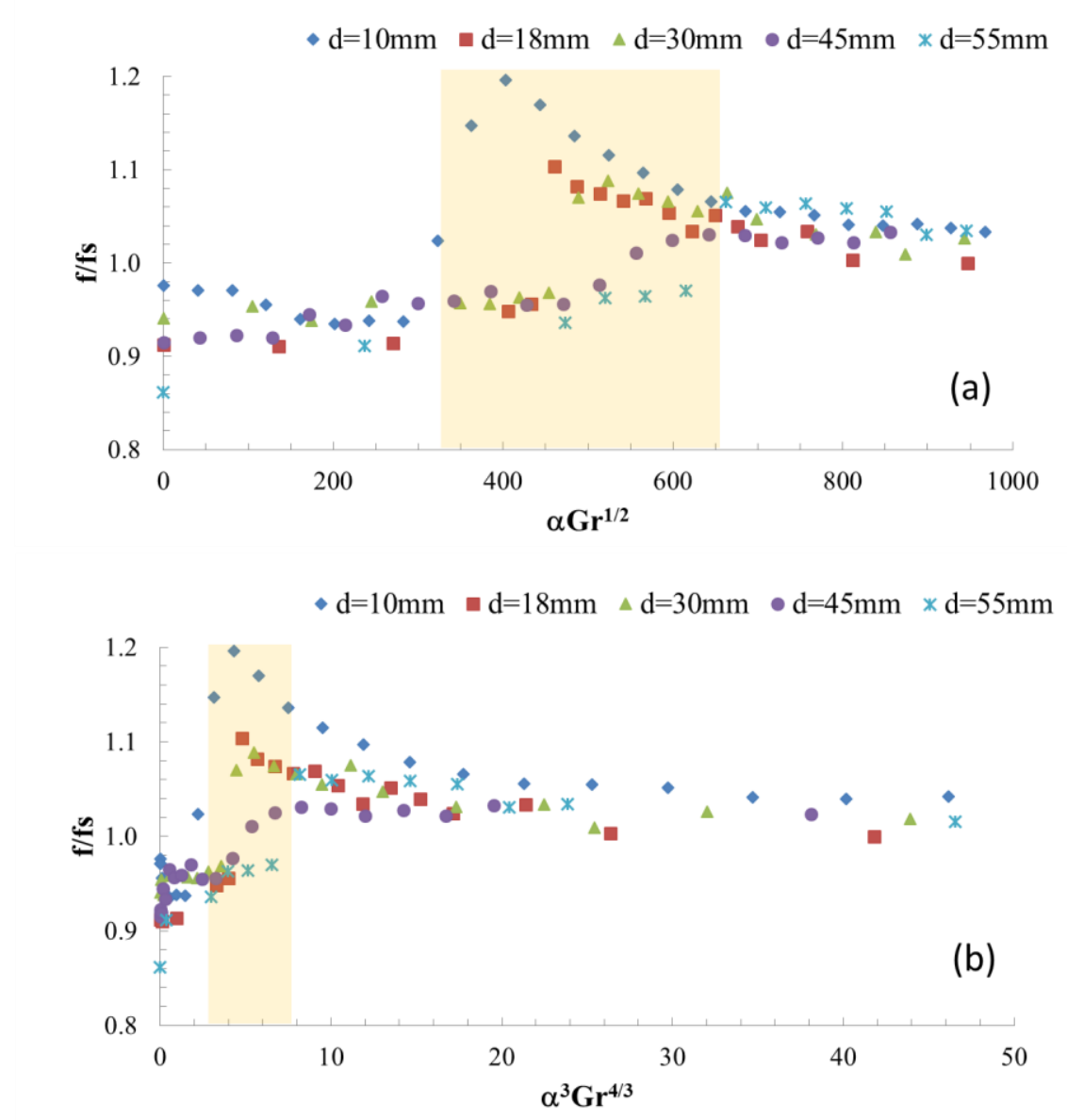

Fig. 9 Nondimensional frequency (by frequency at transition: $f_{\mathrm{s}}$ ) as a function of the characteristic of global parameter (either (a) $\alpha G r^{1 / 2}$ or (b) $\alpha^{3} G r^{4 / 3}$ ). The solid symbols correspond to the experimental data.

Recall that in Yang's work (Yang et al., 2019), a modified Grashof number $\left(\alpha G r^{1 / 2}\right)$ was proposed with a characteristic global parameter, to summarize the transition under the range considered, which is the limited range of the fire scale with limited data (see Fig. 8). In fact, in their report, it was found that their global parameter gave some discrepancy and they explained this via temperature dependency on viscosity. An updated global parameter, $\alpha^{3} G r^{4 / 3}$, works better for a wide range of the transition, and discrepancy shall be minimized. To confirm this, we have attempted to summarize all data available into Fig. 9, using an updated global parameter, and a comparison indicates that the updated parameter works well in revealing the critical condition in a more precise manner.

\section{Conclusion}

A dynamic behavior of interacting two buoyant-jet flames was studied experimentally by varying fuel flowrate, burner diameter and burner separation distance as experimental parameters. Experimental hardware was updated in order to identify the transition of oscillation mode (in-phase and anti-phase) for larger burner cases. Orthogonal flame images 
were additionally taken to confirm the dynamic motion occurring in the 2-D plane. Schlieren imaging was introduced to identify the relationship between the disturbance in a hot plume and the visible flame. Dynamic frequency was measured by a thermocouple signal placed near the burner exit. FFT analyses provided the clear transition condition depending on the adopted experimental parameters. The results confirmed that there is critical condition giving the transition one mode to the other at specific burner separation distance, $L$. This confirmed that burner separation distance is the important length scale in mode transition behavior. It was found that the fuel flowrate $(Q)$ is insensitive to the mode transition, suggesting that the transition is purely governed by buoyancy, not the jet momentum. When a larger burner was used, the frequency "jump" to identify the transition became less pronounced, although we tracked the point clearly for the larger burner case. Importantly it was clearly observed that variation of burner diameter, $d$, was sensitive to the transition point, revealing that the burner diameter is an additional, important length scale. Interestingly we found these two length scales were well-correlated each other, suggesting that there is (at least) one constrained condition connecting their relation. Based on the dimensional analysis, we can successfully propose the updated global parameter to describe the transition as $\alpha^{3} G r^{4 / 3}$, where $\alpha$ and $G r$ stand for the ratio of two length scales $(L / d)$ and Grashof number, respectively. This is somewhat different from the one proposed by Yang et al. (2019) because their prediction is based on the limited range of the fire scale.

\section{Acknowledgements}

This work is supported by the joint scholarship program of Pathumwan Institute of Technology (PIT, Thailand), and Toyohashi University of Technology (TUT, Japan). Technical assistance from, and discussions with, by Mr. T. Yamazaki and former student (Mr. Mochizuki) from TUT are greatly appreciated.

\section{References}

Bunkwang, A. Matsuoka, T. and Nakamura, Y., Mode transition of interacting flickering flames, 27th International Colloquium on the Dynamics of Explosions and Reactive Systems (2019), Beijing, China.

Cetegen, B. M. and Ahmed, T. A., Experiments on the Periodic Instability of Buoyant Plumes and Pool Fires, Combustion and Flame, Vol.93, (1993), pp.157-184.

Cetegen, B. M. and Dong, Y., Experiments on the Instability Modes of Buoyant Diffusion Flames and Effects of Ambient Atmosphere on the Instabilities, Experiments in Fluids, Vol.28, (2000), pp.546-558

Carpio, J., Sanz, S. M. and Tarrazo, F. E., Pinch-off in Forced and Non-forced, Buoyant Laminar Jet Diffusion Flames, Combustion and Flame, Vol.159, (2012), pp.161-169.

Hottel, H. C. and Hawthorne, W. R., Diffusion in Laminar Flame Jets, Third Symposium on Combustion and Flame, and Explosion Phenomena, Vol.3, No.1, (1948), pp.254-266.

Hamins, A., Yang, J. C. and Tashiwaga, T., An Experimental Investigation of the Pulsation Frequency of Flames, TwentyFourth Symposium (International) on Combustion (1992), pp.1695-1702.

Kitahata, H., Taguchi, J., Nagayama, M., Sakurai, T., Ikura, Y., Osa, A., Sumino, Y., Tanaka, M., Yokoyama, E. and Miike, H., Oscillation and Synchronization in the Combustion of Candles, The Journal of Physical Chemistry A, Vol.113, (2009), pp. 8164-8168

Mochizuki, M. and Nakamura, Y., Experimental study on dynamically synchronized behavior of two flickering jet flames, Proc. AOSFST-10 (2015), Tsukuba, Japan, Poster-\#062 (on USB).

Mochizuki, M., Matsuoka, T. and Nakamura, Y., Study on oscillation and transition behavior of interacting flickering flames, Bulletin of Japan Association for Fire Science and Engineering (in Japanese), Vol.67, No.2: 21. (2017).

Toong, T. Y., Salant, R. F., Stopford, J. M. and Anderson, G. Y., Mechanism of Combustion Instability, Tenth Symposium (International) on Combustion (1965), pp.1301-1313.

Xia, X. and Zhang, P., A Vortex-dynamical Scaling Theory for Flickering Buoyant Diffusion Flames, Journal of Fluid Mechanics, Vol.855, (2018), pp.1156-1169.

Yang, T., Xia, X., and Zhang, P., Vortex-dynamical Interpretation of Anti-phase and In-phase Flickering of Dual Buoyant Diffusion Flames, Physical Review Fluids, Vol.4, (2019), 053202. 